\title{
Neuroblastoma versus systemic JIA - a diagnostic dilemma
}

\author{
Lisabeth Scalzi ${ }^{4 *}$, Greg Hychko², Barbara E Ostrov ${ }^{1}$, Catherine A Bingham ${ }^{3}$ David Ungar ${ }^{1}$, Brandt P Groh $^{1}$ \\ From 2011 Pediatric Rheumatology Symposium sponsored by the American College of Rheumatology \\ Miami, FL, USA. 2-5 June 2011
}

\section{Purpose}

Children with malignancies, including neuroblastoma (NB), may present to their primary physician with complaints that mimic those seen in systemic juvenile idiopathic arthritis (sJIA). The purpose of this investgation was to identify significant distinctions between patients with systemic JIA and neuroblastoma at the time of disease presentation.

\section{Methods}

A retrospective chart review was completed on all patients less than age 18, between 1990 and 2009 at Pennsylvania State University Children's Hospital, with a diagnosis of either sJIA or NB. The following variables were identified at the time of presentation; gender, age, race, time to diagnosis, presenting signs and symptoms, neuroblastoma stage, LDH, uric acid, sedimentation rate, CRP, platelet count, hemoglobin, peripheral white blood cell count, percentage of neutrophils and lymphocytes, ferritin, and d-dimer. Other categorical information included whether or not the patients had gait disturbance or extremity pain, fever, rash, abdominal mass, arthritis, fatigue, or weight loss. Individual logistic regression models were evaluated for each variable, with sJIA or NB as the outcome. Given that patients with NB and extremity pain may be referred to orthopedics or rheumatology first, instead of a timely referral to hematology/oncology, a subgroup analysis was completed for those patients.

Table 1 Presentation characteristics of SJIA versus NB patients

\begin{tabular}{llll}
\hline Variables & sJIA (65) & Neuroblastoma (89) & $<.0001$ \\
\hline Age (years) & $7.2 \pm 4.7$ & $3.0 \pm 3.5$ & 0.26 \\
\hline Gender & Famle 51\% & Female 42\% & Male 58\% \\
\hline Race & Male 49\% & White 85\% & 0.86 \\
& White 91\% & Black 7\% & \\
& Black 6\% & Hispanic 3\% & \\
& Hispanic 3\% & Other 5\% & $<0.0001$ \\
\hline Fever present (0=no, 1=yes) & & $12 / 71(18 \%)$ & 0.18 \\
\hline Time to diagnosis (months) & $45 / 45(100 \%)$ & $1.2(78)$ & $<\mathbf{0 . 0 0 0 1}$ \\
\hline Arthritis & $1.5(65)$ & $1 / 71(1 \%)$ & $<\mathbf{0 . 0 0 0 1}$ \\
\hline Rash & $51 / 65(78 \%)$ & $2 / 71(3 \%)$ & 0.0001 \\
\hline Abdominal mass & $53 / 64(83 \%)$ & $29 / 71(41 \%)$ & 0.07 \\
\hline Weight loss & $1 / 65(2 \%)$ & $5 / 71(7 \%)$ & $<0.0001$ \\
\hline Gait disturbance or extremity pain & $11 / 65(17 \%)$ & $13 / 73(18 \%)$ & \\
\hline
\end{tabular}

${ }^{4}$ Pennsylvania State University/Hershey, Hershey, PA, USA

Full list of author information is available at the end of the article

(c) 2012 Scalzi et al; licensee BioMed Central Ltd. This is an Open Access article distributed under the terms of the Creative Commons 
Table 1 Presentation characteristics of SJIA versus NB patients (Continued)

\begin{tabular}{llll}
\hline Neuroblastoma stage & $\mathrm{n} / \mathrm{a}$ & $\begin{array}{l}\text { Stage } 1=19 / 62(31 \%) \\
\text { Stage } 2=3 / 62(5 \%) \\
\text { Stage } 3=9 / 62(14 \%) \\
\text { Stage } 4=31 / 62(50 \%)\end{array}$ \\
\hline LDH (units/L) & $637 \pm 530(35)$ & $1842 \pm 2410(46)$ & $\mathbf{0 . 0 0 5}$ \\
\hline Uric Acid (mg/dL) & $3.3 \pm 1.2(22)$ & $5.1 \pm 2.9(34)$ & $\mathbf{0 . 0 0 5}$ \\
\hline Crp (mg/dL) & $14.9 \pm 25.3(26)$ & $10.2 \pm 7.2(4)$ & 0.71 \\
\hline ESR (mm/hr) & $81.6 \pm 36.3(58)$ & $61.7 \pm 46.0(13)$ & 0.09 \\
\hline PLT count & $464,016 \pm 173,148(62)$ & $327,419 \pm 136,488(62)$ & $<0.00001$ \\
\hline WBC & $18,881 \pm 11,449(63)$ & $10,484 \pm 5,892(68)$ & $<0.00001$ \\
\hline HGB (g.dL) & $10.2 \pm 1.80(62)$ & $10.3 \pm 2.46(67)$ & 0.97 \\
\hline Neurtophil \% & $73 \pm 14(57)$ & $0.49 \pm 0.17(64)$ & $<0.00001$ \\
\hline Lymphocyte \% & $17 \pm 10(47)$ & $40 \pm 17(64)$ & $<0.00001$ \\
\hline Ferntin $(\mathrm{ng} / \mathrm{mL})$ & $4,016 \pm 6,135(50)$ & $159 \pm 205(29)$ & $\mathbf{0 . 0 0 1}$ \\
\hline D-Dimer elevated & $34 / 36(94 \%)$ & $2 / 2(100 \%)$ & 0.73 \\
\hline Aldolase & $15.6 \pm 11.4(28)$ & $8.85 \pm 1.01(2)$ & 0.42
\end{tabular}

\section{Results}

There was a total of 65 sJIA and 89 NB patients. Demographically, sJIA patients were significantly older (7.2 vs. 3.0 years of age; $\mathrm{p}<0.0001)$. Symptomatically, the sJIA patients had fever, rash,arthritis, and extemity pain or gait disturbance ( $41 \%$ vs. $18 \%)$ more frequently $(\mathrm{p}<0.0001$ for all). Laboratories were also significantly different between the groups. NB patients had higher LDH and uric acid levels, while sJIA patients had higher platelet counts, total peripheral WBC, had relative neutrophilia ( $\mathrm{p}<0.0001$ for all), and had higher ferritin levels than the NB patients $(\mathrm{p}=0.001)$, see Table 1 . In the subgroup of 58 children with extremity pain or gait disturbance, 45 (78\%) had sJIA and 13 (22\%) had NB; see Table 2. NB patients had higher LDH $(\mathrm{p}=0.01)$ and lower hgb levels $(p=0.003)$, while sJIA patients had a relative neutrophilia (76\% versus $51 \%$; $\mathrm{p}<0.0001$ ).

Table 2 Laboratory values for sJIA and NB subjects with gait disturbance or extremity pain

\begin{tabular}{llll}
\hline Laboratory & sJIA & NB & p-value \\
\hline LDH & $625 \pm 522(28)$ & $1365 \pm 1073(9)$ & 0.01 \\
Uric Acid & $3.2 \pm 1.1(20)$ & $4.0 \pm 1.2(8)$ & 0.14 \\
CRP & $9.9 \pm 7.8(22)$ & $10.2 \pm 7.3(4)$ & 0.95 \\
ESR & $84 \pm 32(42)$ & $100 \pm 32(6)$ & 0.24 \\
Hgb & $10.4 \pm 1.7(43)$ & $8.5 \pm 2.4(12)$ & $\mathbf{0 . 0 0 3}$ \\
Neutrophil \% & $76 \pm 12(40)$ & $51 \pm 10(11)$ & $<\mathbf{0 . 0 0 0 1}$ \\
Lympocyte \% & $15 \pm 9(40)$ & $37 \pm 12(11)$ & $<\mathbf{0 . 0 0 0 1}$ \\
Ferritin & $4554 \pm 6890(37)$ & $555 \pm 135(3)$ & 0.34 \\
d-dimer abnormal & $27 / 20$ & $1 / 1$ & 0.79 \\
PTT & $35 \pm 6(30)$ & $31 \pm 4(5)$ & 0.15 \\
Aldolase & $16 \pm 2(24)$ & $9 \pm 8(2)$ & 0.44 \\
\hline
\end{tabular}

\section{Conclusion}

There are significantly different demographic, presenting symptoms, and laboratories at the time of presentation of patients who are ultimately diagnosed with sJIA or NB. In particular, there are discerning laboratories that may help facilitate an appropriate and timely referal to oncology when extremity pain or gait disturbance are among the intial signs and symptoms.

\section{Disclosure}

Lisabeth Scalzi: None; Greg Hychko: None; Barbara E. Ostrov: None; Catherine A. Bingham: None; David Ungar: None; Brandt P. Groh: None.

\section{Author details}

${ }^{1}$ Hershey Medical Center, Hershey, PA, USA. ${ }^{2}$ Indiana University, Bloomington, IN, USA. ${ }^{3}$ Zionsville, PA, USA. ${ }^{4}$ Pennsylvania State University/Hershey, Hershey, PA, USA

Published: 13 July 2012

doi:10.1186/1546-0096-10-S1-A33

Cite this article as: Scalzi et al:: Neuroblastoma versus systemic JIA - a

diagnostic dilemma. Pediatric Rheumatology 2012 10(Suppl 1):A33. 